\title{
Long-term Clinical Course of Post-infectious Irritable Bowel Syndrome After Shigellosis: A 10-year Follow-up Study
}

\author{
Young Hoon Youn, ${ }^{1}$ Hyeon Chang Kim, ${ }^{2}$ Hyun Chul Lim, ${ }^{1}$ Jae Jun Park, ${ }^{1}$ Jie-Hyun Kim, ${ }^{1}$ and Hyojin Park ${ }^{1 *}$ \\ ${ }^{\prime}$ Division of Gastroenterology, Department of Internal Medicine, Gangnam Severance Hospital, Yonsei University College of Medicine, Seoul, \\ Korea; and ${ }^{2}$ Department of Preventive Medicine and Public Health, Yonsei University College of Medicine, Seoul, Korea
}

\section{Background/Aims}

A limited number of studies are available regarding the long-term natural history of post-infectious irritable bowel syndrome (PI-IBS). We aimed to investigate the long-term clinical course of PI-IBS.

\section{Methods}

A prospective cohort study was conducted from a 2001 shigellosis outbreak in a Korean hospital with about 2000 employees. A cohort of 124 hospital employees who were infected by Shigella sonnei due to contaminated food in the cafeteria, and 105 sex- and age-matched, non-infected, controls were serially followed for their bowel symptoms by questionnaire surveys for 10 years.

\section{Results}

The Shigella-infected cohort showed significantly higher odds ratio for irritable bowel syndrome (IBS) at 1-year (11.90; 95\% Cl, 1.4995.58) and 3-year (3.93; 95\% Cl, 1.20-12.86) follow-up, compared to their controls. However, corresponding odds ratio for PI-IBS was not significantly increased at 5-year $(1.88 ; 95 \% \mathrm{Cl}, 0.64-5.54)$ and 8 -year $(1.87 ; 95 \% \mathrm{Cl}, 0.62-5.19)$ follow-up. At 10-year followup survey, the prevalence of IBS was similar for the Shigella-infected cohort and their controls $(23.3 \%$ versus $19.7 \%, P=0.703)$. Risk factors which were independently associated with PI-IBS among the Shigella-infected cohort included younger age, previous history of functional bowel disorder, and longer duration of diarrhea at baseline.

\section{Conclusions}

Patients who were infected by Shigella sonnei experienced significantly increased risk of IBS until 3 years after shigellosis, and modestly increased risk until 8 years, but showed similar risk of IBS with uninfected controls at 10 years post-infection. PI-IBS is quite a chronic disorder, and follows a long-term natural course.

\section{(J Neurogastroenterol Motil 2016;22:490-496)}

\section{Key Words}

Cohort; Dysentery; Irritable bowel syndrome; Prognosis; Shigella

\section{Received: October 5, 2015 Revised: January 9, 2016 Accepted: January 27, 2016}

(a) This is an Open Access article distributed under the terms of the Creative Commons Attribution Non-Commercial License (http://creativecommons. org/licenses/by-nc/4.0) which permits unrestricted non-commercial use, distribution, and reproduction in any medium, provided the original work is properly cited.

*Correspondence: Hyojin Park, MD, PhD 


\section{Introduction}

Irritable bowel syndrome (IBS) is one of most common chronic gastrointestinal disorders, and is characterized by abdominal pain or discomfort associated with disturbed and altered bowel habit in the absence of organic disease. ${ }^{1}$ IBS is a common digestive disease, with a variable prevalence of $4-22 \%$ worldwide. ${ }^{2}$ Although it is not a lifethreatening disorder, IBS poses significant quality of life concerns and remains a substantial burden on the health care system. ${ }^{3}$

The pathophysiology of IBS is thought to be multifactorial, and possible mechanisms of IBS include altered bowel motility, visceral hypersensitivity, psychosocial distress, abnormal brain-gut interaction, enteric infection, gut-immune activation, low grade inflammation, intestinal permeability, and alterations in intestinal microflora. ${ }^{4-9}$

The role of enteric infection in the pathogenesis of IBS has been recognized since Chaudhary and Truelove ${ }^{10}$ first reported the association between infectious gastroenteritis and persistent gastrointestinal symptoms. While many enteric infections cause selflimiting illness, 5-30\% of people experience new gastrointestinal symptoms that persist after bacterial dysentery, despite clearance of the inciting pathogen. ${ }^{11}$ Additionally, approximately $10 \%$ of IBS patients reported that their symptoms began following a bout of infectious dysentery. ${ }^{12}$

Post-infectious IBS (PI-IBS) is defined as the acute onset of new IBS symptoms in an individual who has not previously met the criteria for IBS immediately following an acute illness characterized by 2 or more of the following: fever, vomiting, diarrhea, or positive bacterial stool culture. ${ }^{12}$

Although there have been many reports regarding PI-IBS associated with pathogens such as Shigella spp., pathogenic Escherichia coli, Salmonella, Campylobacter jejuni, and Giardia duodenalis in the past 20 years, ${ }^{13-19}$ most of these reports address the shortterm clinical course of PI-IBS, and only few examined the longterm course ( $\geq 5$ years) of PI-IBS. ${ }^{20-23}$

We previously reported on the clinical course of PI-IBS in a homogenous cohort comprised of patients recovered from shigellosis. ${ }^{17,23,24}$ In the current study, we report on the collective results of a 10-year, long-term follow-up of our small, well-followed, homogenous cohort of patients that developed PI-IBS after shigellosis.

\section{Materials and Methods}

\section{Subjects}

In December 2001, a shigellosis outbreak took place at Gangnam Severance Hospital (formerly "Youngdong Severance Hospital") in Seoul, Korea, due to the consumption of contaminated food in the hospital employee cafeteria. The causative pathogen was Shigella sonnei, and a total of 181 patients were diagnosed and treated for shigellosis. All patients were medical personnel including physicians, nurses, paramedics, and medical students. After termination of the shigellosis outbreak, we established a cohort of Shigella-exposed patients who agreed to participate in serial follow-up surveys. Patients who were pregnant, those who had already symptoms of IBS before shigellosis, and who had a history of abdominal surgery or chronic organic gastrointestinal disorders were excluded. The initial Shigella-exposed cohort was comprised of 143 subjects.

To serve as a control population, we recruited healthy ageand sex-matched volunteers who were working for the hospital at the time of the shigellosis outbreak (they were not exposed to the contaminated food), and among them, subjects who already had symptoms of IBS before shigellosis was excluded from control cohort and followed separately as sporadic IBS subjects in subsequent analyses.

For the 10-year follow-up survey, we built a list of 124 subjects in the Shigella-exposed cohort who were still working for the hospital, or those who were retired but contactable by phone calls. Among the control group, we were able to contact 105 subjects in the same manner.

\section{Methods}

Both the Shigella-exposed cohort and the control cohort were asked to participate in follow-up questionnaires at 1, 3, 5, 8, and 10 years after recruitment. The initial 1-year and 3-year surveys addressed epidemiologic data, past medical and surgical history, information regarding the presence of vomiting, fever, and duration of diarrhea during the acute illness, and information regarding current bowel symptoms according to the Rome II criteria. ${ }^{1724}$ The followup questionnaires at 5, 8, and 10 years after outbreak mainly addressed current bowel symptoms according to the Rome II criteria, as well as recent medical history of other gastrointestinal disorders, including incidence of additional enteric infection and/or abdominal surgery, which was exclusion criteria for subsequent analyses. ${ }^{23}$ The diagnosis of IBS and other functional bowel disorders (FBDs) was 
made using the Rome II criteria, and other FBDs included functional abdominal bloating, functional constipation, functional diarrhea, and unspecified FBDs

For the 10-year follow-up survey, investigators performed the survey questionnaire preferentially by face-to-face interview, and by telephone interview, if necessary. A small financial reward (\$10) was given in acknowledgement of subjects' participation in the study. Written informed consent was obtained from each subject before the survey, and the University of Yonsei Medical School Ethics Committee approved the study protocol (IRB approval number: 3-2011-0242).

\section{Statistical Methods}

Cohort members and their controls were compared by independent $t$ test for continuous variables, and by Chi-square test or Fisher's exact test for categorical variables. Logistic regression analysis was performed to calculate $\mathrm{OR}$ and $95 \% \mathrm{CI}$ for prevalent IBS at each time interval or newly-developed IBS during a given timeinterval, after adjustment for sex and age. All statistical calculations were performed using the Statistical Package for the Social Sciences (SPSS, 18.0 Inc, Chicago, IL, USA). A P-value of less than 0.05 was regarded as statistically significant.

\section{Results}

\section{Response Rate and General Characteristics}

A total of 229 subjects (124 in the Shigella-exposed group, 105 in the control group) were surveyed periodically for 10 years following the December 2001 shigellosis outbreak. Ten years postoutbreak, the overall response rate was $70.7 \%$ (162/229), while the response rate of the Shigella-exposed group was $69.4 \%$, which was not significantly different from the response rate $(72.4 \%)$ of the control group (Table 1). The mean age of study subjects 10 years post-outbreak was 41.8 years for the Shigella-exposed group and 39.6 years for the control group $(P=0.071)$. The female-to-male ratio was 2.8 for the Shigella-exposed group and 2.9 for the control group $(P=0.943)$.

\section{Prevalence of Irritable Bowel Syndrome at Each Time Point}

The prevalence of IBS after the shigellosis outbreak was analyzed at each time point after exclusion of subjects who had symptoms of IBS prior to the outbreak (Table 2).

The prevalence of IBS after 1 year was $13.1 \%$ in the Shigellaexposed group and $1.1 \%$ in the control group. Thus, the risk of IBS

Table 1. Survey Response Rate at Each Time Point

\begin{tabular}{|c|c|c|c|c|}
\hline \multirow{2}{*}{ Survey time (yr) } & \multicolumn{2}{|c|}{ Shigella-exposed group } & \multicolumn{2}{|r|}{ Control group } \\
\hline & Planned subjects & Responded subjects (response rate) & Planned subjects & Responded subjects (response rate) \\
\hline 1 st & 143 & $101(70.6 \%)$ & 113 & $102(90.3 \%)$ \\
\hline $3 \mathrm{rd}$ & 124 & $95(76.6 \%)$ & 120 & $105(87.5 \%)$ \\
\hline 5 th & 75 & $59(78.7 \%)$ & 78 & $60(76.9 \%)$ \\
\hline 8 th & 133 & $71(53.4 \%)$ & 105 & $65(61.9 \%)$ \\
\hline 10 th & 124 & $86(69.4 \%)$ & 105 & $76(72.4 \%)$ \\
\hline
\end{tabular}

Table 2. Prevalence of Newly-developed Irritable Bowel Syndrome After Shigellosis Outbreak at Each Time Point

\begin{tabular}{|c|c|c|c|c|c|}
\hline \multirow{2}{*}{ Survey time (yr) } & \multicolumn{2}{|c|}{ Shigella-exposed group } & \multicolumn{2}{|c|}{ Control group } & \multirow{2}{*}{$\begin{array}{l}\text { Adjusted OR } \\
\quad(95 \% \mathrm{CI})\end{array}$} \\
\hline & Total No. & No. of IBS & Total No. & No. of IBS & \\
\hline $1 \mathrm{st}$ & 87 & $12(13.8 \%)$ & 89 & $1(1.1 \%)$ & $11.9(1.49-95.58)$ \\
\hline $3 \mathrm{rd}$ & 87 & $13(14.9 \%)$ & 89 & $4(4.5 \%)$ & $3.93(1.20-12.86)$ \\
\hline 5 th & 53 & $11(20.8 \%)$ & 49 & $6(12.2 \%)$ & $1.88(0.64-5.54)$ \\
\hline 8th & 71 & $11(15.4 \%)$ & 65 & $6(9.2 \%)$ & $1.87(0.62-5.19)$ \\
\hline 10th & 86 & $20(23.3 \%)$ & 76 & $15(19.7 \%)$ & $1.61(0.70-3.69)$ \\
\hline
\end{tabular}

IBS, irritable bowel syndrome. 
in the Shigella-exposed cohort was significantly higher than that of the control cohort at the 1-year follow-up survey (adjusted OR, 11.90). The prevalence of IBS at the 3-year follow-up survey was also significantly higher in the Shigella-exposed group than the control group (adjusted OR, 3.93). However, at the 5-year and 8-year follow-up surveys, the prevalence of IBS in the Shigella-exposed cohort was modestly increased but not statistically significant compared to the control cohort. The 10-year follow-up survey showed similar IBS prevalence in both groups $(23.3 \%$ for Shigella-exposed group, $19.7 \%$ for control group; $P=0.703$ ).

\section{Incidence of Irritable Bowel Syndrome During Each Time Interval}

The incident of IBS during each time interval was defined as newly-developed IBS among subjects who did not report IBS symptoms in the previous survey (Table 3). The incidence of newlydeveloped IBS was significantly higher in the Shigella-exposed group than the control group during the first year post-outbreak. However, no significant differences were demonstrated between the 2 groups at subsequent follow-up intervals.

\section{Irritable Bowel Syndrome Prognosis}

We were able to follow-up 12 patients who developed PI-IBS among the Shigella-exposed cohort up to 10 years after the shigellosis outbreak. Also, we could follow up the natural history of 14 sporadic IBS (not "post-infectious") patients among the control cohort who were initially recruited at the time of outbreak, ${ }^{23}$ and were excluded from the subsequent analysis of newly-developed IBS because they already had symptoms of IBS prior to the outbreak.

The figure represents the 10-year natural history of PI-IBS patients (Figure A) and sporadic IBS patients (Figure B). About half of the PI-IBS patients showed remission of IBS symptom after 5 years. However, approximately $25-30 \%$ of PI-IBS patients had persistent IBS symptoms even after 8 to 10 years. The natural his-

Table 3. Incidence of Newly-developed Irritable Bowel Syndrome During Each Time Interval

\begin{tabular}{ccccccc}
\hline \multirow{2}{*}{ Time interval (yr) } & \multicolumn{2}{c}{ Shigella-exposed group } & & \multicolumn{2}{c}{ Control group } & \multicolumn{2}{c}{$\begin{array}{c}\text { Adjusted OR } \\
(95 \% \text { CI })\end{array}$} \\
\cline { 2 - 3 } & Total No. & No. of incident IBS & & Total No. & No. of incident IBS & $11.9(1.49-95.58)$ \\
$0-1$ & 87 & $12(13.8 \%)$ & & 89 & $1(1.1 \%)$ & $1.61(0.35-7.46)$ \\
$1-3$ & 71 & $4(5.6 \%)$ & & 84 & $3(3.6 \%)$ & $2.15(0.50-9.18)$ \\
$3-5$ & 46 & $6(13.0 \%)$ & & 46 & $3(6.5 \%)$ & $1.23(0.26-5.73)$ \\
$5-8$ & 71 & $4(5.6 \%)$ & & 65 & $3(4.6 \%)$ & $1.04(0.47-2.28)$ \\
\hline-10 & 71 & $11(15.5 \%)$ & & 67 & $10(14.9 \%)$ & \\
\hline
\end{tabular}

IBS, irritable bowel syndrome.
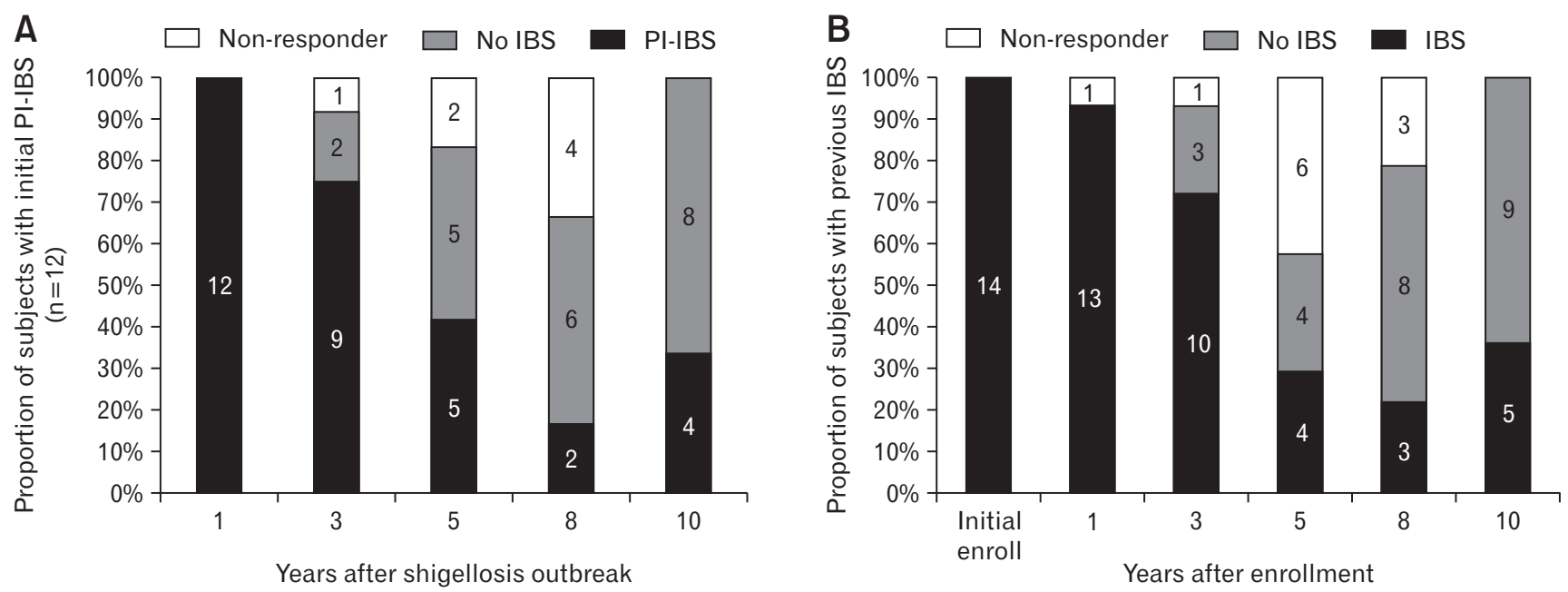

Figure. Natural history of post-infectious irritable bowel syndrome (PI-IBS) after shigellosis (A), and sporadic IBS in control subjects (B) over 10 years. Twelve PI-IBS patients and 14 sporadic IBS patients were followed for 10 years. Approximately half the patients recovered from IBS after 5 years, but several patients had persistent IBS symptoms for the duration of the study. The natural history of PI-IBS and sporadic IBS seem to be quite similar. 
Table 4. Analysis of Risk Factors Associated with Development of Post-infectious Irritable Bowel Syndrome in Shigella-exposed Group During 10-year Follow-up

\begin{tabular}{ll}
\hline Baseline characteristics & OR $(95 \% \mathrm{CI})$ \\
\hline Age $(\mathrm{yr})$ & 1.00 \\
$\quad<40$ & $0.94(0.89-0.99)$ \\
$\geq 40$ & 1.00 \\
Sex & $2.00(0.75-5.54)$ \\
$\quad$ Male & \\
Female & 1.00 \\
History of other FBD & \\
No & $5.10(1.2-21.1)$ \\
Yes & 1.00 \\
Duration of diarrhea & $1.27(1.01-1.60)$ \\
No & \\
Yes & 1.00 \\
Vomiting & $0.58(0.14-2.39)$ \\
No & \\
Yes & 1.00 \\
Fever & $1.57(0.68-3.64)$ \\
No & \\
Yes &
\end{tabular}

${ }^{\mathrm{a}}$ Functional bowel disorders (FBDs) other than irritable bowel syndrome (IBS), including functional abdominal bloating, functional constipation, functional diarrhea, and unspecified FBDs by Rome II criteria.

tory of the 14 sporadic IBS (not-related to shigellosis) patients was quite similar to the prognosis of PI-IBS patients.

\section{Risk Factors Associated with Post-infectious Irritable Bowel Syndrome Development}

We analyzed the risk factors which were associated with the ever-developed PI-IBS in the Shigella-exposed subjects. Younger age, previous history of FBDs other than IBS at baseline, and duration of diarrhea in initial dysentery were significantly associated with PI-IBS development during the follow-up over 10 years (Table 4).

\section{Discussion}

Since Chaudhary and Truelove ${ }^{10}$ first reported the concept of PI-IBS in 1962, there has been a substantial amount of epidemiologic data published regarding the association between PI-IBS and various pathogens, including bacterial, protozoal, and viral pathogens. Several studies have established the causative pathogens of PI-IBS, including Shigella spp., ${ }^{16,23}$ pathogenic E. coli, ${ }^{22}$ Salmonella spp. ${ }^{13,18}$ C. jejuni, ${ }^{15}$ G. duodenali, ${ }^{19}$ Trichinella britovi, ${ }^{25}$ norovirus, ${ }^{26}$ or combinations of the preceding pathogens. ${ }^{14,22}$

The reported incidence of PI-IBS varies from about $5-30 \%,{ }^{11}$ and PI-IBS is now a well-recognized consequence of acute infectious gastroenteritis. There is little doubt that bouts of infectious enteritis may cause some patients to suffer from chronic IBS symptoms, and this fact was also validated by the results of our study. However, little data is available for long-term natural history of PIIBS.

As far as we know, there have been only 3 studies that have followed participants with PI-IBS for more than 5 years. ${ }^{20-22}$ Among these relatively long-term reports, one is a 6-year follow-up study from a British cohort, ${ }^{20}$ another is a 5 -year follow-up study from a Swedish cohort, ${ }^{21}$ and the other is an 8-year follow-up study from a Canadian cohort. ${ }^{22}$ However, our study followed Korean patients for 10 years, and as such is the longest follow-up study concerning PI-IBS. Moreover, our cohort consisted of a homogenous group of patients who were infected with a single pathogenic strain $(S$. sonnei) from the same contamination source. The 8-year followup study with thousands of participants from Walkerton, Canada ${ }^{22}$ could provide the most useful data due to the sample size and consequential statistical power, while our study is limited in its small sample size. However, in the Walkerton cohort, the infecting pathogens were variable combinations of E. coli 0157:H7, C. jejuni, and other pathogens, ultimately resulting in increased heterogeneity of the study population.

Our current study might improve our understanding of longterm prognosis of PI-IBS. Approximately half of the patients in our study recovered 5 years post-infection, whereas $25-33 \%$ still suffered from PI-IBS even 8-10 years post-infection. These results are generally consistent with those of previous long-term studies. ${ }^{20-22}$ Additionally, we found that the long-term natural course of PIIBS was similar to that of sporadic IBS in our cohort. Although we found a slightly higher incidence of IBS in the Shigella-exposed group than the control group at 5 and 8 years post-outbreak, the differences were not statistically significant. These findings are in contrast with those of the Walkerton study, where the difference in IBS prevalence was still significantly higher after 8 years in the infected group compared to the control group (OR, 3.12; 95\% CI, 1.99-5.04). ${ }^{22}$ This discrepancy might have resulted from the difference in sample size, infecting pathogens, or population characteristics. With this in mind, the non-significant difference in IBS prevalence between the infected and control groups in our study after 5 years does not indicate that acute infectious gastroenteritis did not affect the PI-IBS patients for longer than 5 years. The issue of how long the difference in the prevalence of IBS lasts between infected and control group cannot be clearly concluded yet, and we believe that this issue might be also influenced by regional socioenvironmental factors of the each cohort. 
The risk of newly-developing PI-IBS in the Shigella-exposed group is significantly higher only during the first year post-infection, whereupon the development of new sporadic IBS was the same in the 2 groups. Because PI-IBS is a chronic disorder, the difference in the prevalence of IBS between the 2 groups can persist for several years post-infection. After 10 years, however, IBS prevalence in the infected group decreased to nearly the same level as that of the control group. This is likely because the PI-IBS patients recovered gradually while patients in the control group developed new sporadic IBS.

The mechanisms that underlie PI-IBS remain largely unclear, but several mechanisms have been proposed including immune activation, chronic low grade inflammation, enterochromaffin cell hyperplasia, and alteration of epithelial permeability and neuromuscular function. ${ }^{12,27}$ Additionally, functional gastrointestinal disorders following acute infectious gastroenteritis not only include PI-IBS, but also post-infectious dyspepsia. ${ }^{18,28,29}$ The final symptom complex depends on the interaction between local mucosal injury and central factors including adverse life events, personality, and external stressors. Additionally, local injury is often more highly influenced by the nature of the infectious agent. Rotavirus and Giardia infection mainly involve the upper gastrointestinal tract, and thus may predominantly predispose patients to dyspepsia, whereas Shigella infection is confined to the left colon and exclusively predisposes patients to IBS. Salmonella or Campylobacter infections cause ileitis and colitis, equally predisposing patients to both dyspepsia and IBS Because our cohort was comprised of patients exclusively infected by $S$. sonnei, the absence of post-infectious dyspepsia was expected. Conversely, post-infectious dyspepsia was common in the Walkerton cohort. ${ }^{28}$

Risk factors for PI-IBS in the results of our study are consistent with those from previously published studies, which suggest that duration and severity of initial illness, toxicity of infecting pathogens, female sex, younger age, previous history of functional gastrointestinal disorder, and psychosocial factors are risk factors for developing PI-IBS. In our study, younger age, previous history of FBD, and duration of diarrhea showed statistically significant associations with PI-IBS development, whereas female sex and fever showed increased but statistically nonsignificant odds ratios, possibly due to the small number of study subjects. Additionally, we found no association between vomiting and PI-IBS, which may be explained by the nature of Shigella (which primarily affects the left colon).

We followed our cohort with regular surveys on bowel symptoms $1,3,5,8$, and 10 years after outbreak. During the course of the surveys, the response rate and final number of participants were lowest at the 5-year survey, and gradually rose to about $70 \%$ at the 10 -year survey. The small number of participants at the time of the 5 -year survey might be due to the exclusion of all subjects who had been excluded from 1-year and 3-year analysis. The response rate of the 10-year survey was relatively higher than previous surveys, because we tried multiple contacts using both face-to-face interview and telephone interview.

The prevalence of IBS increased between 8-10 years postoutbreak in both the infected and control groups. The cumulative incidence of IBS between 8 to 10 years post-outbreak was $15.5 \%$ in the infected group and $14.9 \%$ in the control group, which are values quite high compared with other interval periods. We cannot definitively explain this increased IBS rates, but we posit that hospital workers may have been exposed to a stressful work environment during that period. Indeed, a series of hospital qualifying programs (Joint Commission International accreditation and Korean Institute for Healthcare accreditation) continued to proceed at the time of the 10th year survey, and the workload of many hospital workers was heavier than usual. We presume that stressful workload might be associated with increase of IBS at the 10th year survey.

The current study has also several limitations. Firstly, the relatively small size of cohorts may decrease the statistical reliability. Secondly, we tried to exclude new PI-IBS due to additional enteric infection after the initial bout of shigellosis, by surveying the history of additional enteric infection between the periods, however there might be some recall bias. Thirdly, the diagnosis of IBS was made by symptoms at only each time points of the surveys, and was not continuously monitored throughout the 10 years, and so, time dependent Cox regression analysis could not be applied to clarify the prognosis of PI-IBS.

In conclusion, the relative risk of PI-IBS was significantly higher in the infected group compared to the control group up to 3 years after shigellosis, but gradually decreased thereafter. The natural history of PI-IBS and sporadic IBS seem to be similar, and in our study approximately half of patients in each group recovered after 5 years, whereas symptoms persisted for 10 years in about one third of patients. The duration of diarrhea as an index of severity of initial illness, previous history of FBD, and younger age were risk factors for PI-IBS.

Financial support: This study was financially supported by the Jeil grant of the Korean Society of Neurogastroenterolgy and Motility (KSNM) for 2011 (KSNM 2011-Jeil), which was donated by Jeil Pharmaceutical company in Korea. The funders had no role in study design, data acquisition and analysis, and interpretation of the results. 


\section{Conflicts of interest: None.}

Author contributions: Young Hoon Youn analyzed data and wrote the manuscript; Hyeon Chang Kim contributed on data analysis, statistic, and revision of manuscript; Hyun Chul Lim, Jae Jun Park, and Jie-Hyun Kim contributed on patients interview and data acquisition; and Hyojin Park designed and supervised this study.

\section{References}

1. Drossman DA. The functional gastrointestinal disorders and the Rome III process. Gastroenterology 2006;130:1377-1390.

2. Gwee KA, Lu CL, Ghoshal UC. Epidemiology of irritable bowel syndrome in Asia: something old, something new, something borrowed. J Gastroenterol Hepatol 2009;24:1601-1607.

3. Nellesen D, Yee K, Chawla A, Lewis BE, Carson RT. A systematic review of the economic and humanistic burden of illness in irritable bowel syndrome and chronic constipation. J Manag Care Pharm 2013;19:755764.

4. McKee DP, Quigley EM. Intestinal motility in irritable bowel syndrome: is IBS a motility disorder? Part 2. Motility of the small bowel, esophagus, stomach, and gall-bladder. Dig Dis Sci 1993;38:1773-1782.

5. Mertz H, Morgan V, Tanner G, et al. Regional cerebral activation in irritable bowel syndrome and control subjects with painful and nonpainful rectal distention. Gastroenterology 2000;118:842-848.

6. Chadwick VS, Chen W, Shu D, et al. Activation of the mucosal immune system in irritable bowel syndrome. Gastroenterology 2002;122:17781783.

7. Azpiroz F, Bouin M, Camilleri M, et al. Mechanisms of hypersensitivity in IBS and functional disorders. Neurogastroenterol Motil 2007;19(1 suppl):62-88.

8. Gwee KA. Post-infectious irritable bowel syndrome, an inflammationimmunological model with relevance for other IBS and functional dyspepsia. J Neurogastroenterol Motil 2010;16:30-34.

9. Dupont HL. Review article: evidence for the role of gut microbiota in irritable bowel syndrome and its potential influence on therapeutic targets. Aliment Pharmacol Ther 2014;39:1033-1042.

10. Chaudhary NA, Truelove SC. The irritable colon syndrome. A study of the clinical features, predisposing causes, and prognosis in 130 cases. Q J Med 1962;31:307-322.

11. Thabane M, Kottachchi DT, Marshall JK. Systematic review and metaanalysis: the incidence and prognosis of post-infectious irritable bowel syndrome. Aliment Pharmacol Ther 2007;26:535-544.

12. Spiller R, Garsed K. Postinfectious irritable bowel syndrome. Gastroenterology 2009;136:1979-1988.

13. McKendrick MW, Read NW. Irritable bowel syndrome--post salmonella infection. J Infect 1994;29:1-3.

14. Neal KR, Hebden J, Spiller R. Prevalence of gastrointestinal symptoms six months after bacterial gastroenteritis and risk factors for development of the irritable bowel syndrome: postal survey of patients. BMJ 1997;314:779-782.

15. Dunlop SP, Jenkins D, Neal KR, Spiller RC. Relative importance of enterochromaffin cell hyperplasia, anxiety, and depression in postinfectious IBS. Gastroenterology 2003;125:1651-1659.

16. Wang LH, Fang XC, Pan GZ. Bacillary dysentery as a causative factor of irritable bowel syndrome and its pathogenesis. Gut 2004;53:10961101.

17. Ji S, Park H, Lee D, Song YK, Choi JP, Lee SI. Post-infectious irritable bowel syndrome in patients with Shigella infection. J Gastroenterol Hepatol 2005;20:381-386.

18. Mearin F, PérezOliveras M, Perelló A, et al. Dyspepsia and irritable bowel syndrome after a Salmonella gastroenteritis outbreak: one-year follow-up cohort study. Gastroenterology 2005;129:98-104.

19. Dizdar V, Gilja OH, Hausken T. Increased visceral sensitivity in Giardiainduced postinfectious irritable bowel syndrome and functional dyspepsia. Effect of the $5 \mathrm{HT}_{3}$-antagonist ondansetron. Neurogastroenterol Motil 2007;19:977-982.

20. Neal KR, Barker L, Spiller RC. Prognosis in post-infective irritable bowel syndrome: a six year follow up study. Gut 2002;51:410-413.

21. Törnblom H, Holmvall P, Svenungsson B, Lindberg G. Gastrointestinal symptoms after infectious diarrhea: a five-year follow-up in a Swedish cohort of adults. Clin Gastroenterol Hepatol 2007;5:461-464.

22. Marshall JK, Thabane M, Garg AX, Clark WF, Moayyedi P, Collins SM. Eight year prognosis of postinfectious irritable bowel syndrome following waterborne bacterial dysentery. Gut 2010;59:605-611.

23. Jung IS, Kim HS, Park H, Lee SI. The clinical course of postinfectious irritable bowel syndrome: a five-year follow-up study. J Clin Gastroenterol 2009;43:534-540

24. Kim HS, Kim MS, Ji SW, Park H. [The development of irritable bowel syndrome after Shigella infection: 3 year follow-up study.] Korean J Gastroenterol 2006;47:300-305. [Korean]

25. Soyturk M, Akpinar H, Gurler O, et al. Irritable bowel syndrome in persons who acquired trichinellosis. Am J Gastroenterol 2007;102:10641069.

26. Marshall JK, Thabane M, Borgaonkar MR, James C. Postinfectious irritable bowel syndrome after a food-borne outbreak of acute gastroenteritis attributed to a viral pathogen. Clin Gastroenterol Hepatol 2007;5:457460.

27. Song J, Zhang L, Bai T, Qian W, Li R, Hou X. Mast cell-dependent mesenteric afferent activation by mucosal supernatant from different bowel segments of guinea pigs with post-infectious irritable bowel syndrome. J Neurogastroenterol Motil 2015;21:236-246.

28. Ford AC, Thabane M, Collins SM, et al. Prevalence of uninvestigated dyspepsia 8 years after a large waterborne outbreak of bacterial dysentery: a cohort study. Gastroenterology 2010;138:1727-1736.

29. Pike BL, Porter CK, Sorrell TJ, Riddle MS. Acute gastroenteritis and the risk of functional dyspepsia: a systematic review and meta-analysis. Am J Gastroenterol 2013;108:1558-1563. 